\title{
Consumo de alcohol y caracterización de los bebedores problemáticos en Galicia
}

\section{Alcohol consumption and characterization of hazardous drinkers in Galicia}

\author{
Julia Rey-Brandariz*, Mónica Pérez-Ríos*, María Isolina Santiago-Pérez**, Beatriz Fontela*, \\ Alberto Malvar**, Xurxo Hervada**. \\ * Área de Medicina Preventiva y Salud Pública. Universidad de Santiago de Compostela. Santiago de Compostela. España. \\ ** Subdirección de Información sobre Saúde e Epidemioloxía. Dirección Xeral de Saúde Pública. Consellería de Sanidade. \\ Xunta de Galicia. Santiago de Compostela. España.
}

\section{Resumen}

Conocer en detalle la epidemiología del consumo de alcohol a nivel poblacional es indispensable para diseñar programas de Salud Pública eficaces. Los objetivos de este estudio fueron estimar la prevalencia del consumo de alcohol diario, ocasional, esporádico, intensivo y problemático entre los gallegos de 16 y más años y caracterizar a los bebedores problemáticos teniendo en cuenta la perspectiva de género. Se realizó un estudio transversal en 2017 entre la población mayor de 15 años residente en Galicia en el marco del Sistema de Información sobre Conductas de Riesgo (SICRI). En 2017, se entrevistaron 7.841 adultos seleccionados mediante muestreo aleatorio estratificado. Se incluyeron preguntas relacionadas con el consumo de alcohol en el último mes y se estimó la prevalencia de consumo problemático empleando el test AUDIT. Para caracterizar a los bebedores problemáticos se ajustó un modelo de regresión logística multivariante. El 18,8\% de la población gallega consumía alcohol a diario y el 3,4\% tenían un consumo problemático. Las prevalencias de consumo de alcohol diario, ocasional, esporádico, intensivo o problemático fueron más altas entre los hombres y variaron por edad. La prevalencia de consumo diario-ocasional y esporádico fue más alta a edades medias (45-64 años), y el intensivo y problemático entre los 16 y 24 años. Las características asociadas a un consumo problemático de alcohol varían por sexo, identificándose características comunes como la edad o ser fumador de tabaco o cannabis, y diferenciales como el nivel de estudios. Las tipologías de consumo cambian en función de la edad y del sexo. Los sistemas de vigilancia deberían monitorizar de forma periódica el consumo de alcohol para adaptar las medidas de prevención a cambios en la tipología de consumo. Palabras clave: consumo de alcohol; prevalencia; transversal; alcoholismo; sexo.

\section{Abstract}

Detailed knowledge of the epidemiology of alcohol consumption at the population level is essential to the design of effective public health programs. The objectives of this study were to estimate the prevalence of daily, sporadic, occasional, binge and hazardous alcohol consumption among the Galician population aged 16 years and over, and to characterize hazardous drinkers, taking into account the gender perspective. A cross-sectional study was conducted in 2017 among people aged over 15 years residing in Galicia in the framework of the Risk Behavior Information System (SICRI). In 2017, 7,841 adults were interviewed by stratified random sampling. Questions related to alcohol consumption in the last month were included and the prevalence of hazardous consumption was estimated using the AUDIT test. A multivariate logistic regression model was fitted to characterize hazardous drinkers. A total of $18.8 \%$ of the Galician population consumed alcohol daily and $3.4 \%$ had hazardous consumption. The prevalence of daily, occasional, sporadic, binge and hazardous drinking was higher among men and varied by age. The prevalence of daily-occasional and sporadic drinking was highest at middle age (45-64 years), and binge and hazardous drinking among those aged 16 to 24 years. The characteristics associated with hazardous drinking varied by gender, and are identified by common characteristics to both genders such as age or being a tobacco or cannabis smoker, and differential characteristics such as educational level. Typologies of consumption changed according to age and gender. Surveillance systems should regularly monitor alcohol consumption in order to adapt prevention measures to changes in drinking patterns.

Key words: alcohol use; prevalence; cross-sectional; alcoholism; gender.

Recibido: Septiembre 2020; Aceptado: Mayo 2021.

Enviar correspondencia a: Mónica Pérez Ríos. Departamento de Medicina Preventiva y Salud Pública, Universidad de Santiago de Compostela, Santiago de Compostela, España. Teléfono-Fax: 0034881812277.

E-mail: monica.perez.rios@usc.es 
$\mathrm{E}$ 1 consumo de alcohol produce aproximadamente el $5 \%$ de la carga de morbilidad y de mortalidad en el mundo (Organización Mundial de la Salud, 2018; Peacock et al., 2018). En 2016, el $6,8 \%$ de las muertes en hombres y el $2,2 \%$ en mujeres a nivel mundial se asociaron con el consumo de alcohol (GBD 2016 Alcohol Collaborators, 2018). El consumo de alcohol está relacionado con más de 200 patologías y aumenta el riesgo de padecer enfermedades como la cirrosis o el cáncer hepático (Organización Mundial de la Salud, 2018; Rehm et al., 2017), y es causa de hospitalización directa y de aumento de la estancia hospitalaria por causa indirecta (Sánchez Sánchez, Redondo Martín, García Vicario y Velázquez Miranda, 2012). Además, está relacionado con los accidentes de tráfico, las conductas sexuales de riesgo, la violencia, el maltrato o la pérdida de productividad laboral (Ministerio de Sanidad, Consumo y Bienestar Social, 2020; Pulido et al., 2014). Todo esto origina grandes costes sanitarios, económicos y sociales y lo sitúa como un importante problema de Salud Pública (Barrio, Reynolds, García-Altés, Gual y Anderson, 2017; Łyszczarz, 2019).

El espectro del consumo de alcohol es amplio y comprende diferentes tipologías, desde la abstinencia o no consumo, hasta la dependencia alcohólica o alcoholismo (Maisto y Saitz, 2003). En España, la Encuesta sobre Alcohol y otras Drogas (EDADES) es la fuente que aporta la información más detallada sobre el consumo de alcohol, si bien se restringe a población de 15 a 64 años. La última encuesta realizada en 2019-2020 estimó para España una prevalencia de consumo de alcohol en el último mes del 63,0\%, y de consumo diario del 8,8\% (Observatorio Español de las Drogas y las Adicciones, 2021). Los datos más recientes en población general adulta (15 y más años) derivan de la Encuesta Nacional de Salud realizada en 2017 (ENSE-2017). En ella se estima que la prevalencia de consumidores diarios de alcohol es del 14,7\%, siendo Galicia la sexta comunidad autónoma con la prevalencia de consumo más elevada (17,3\%) (Ministerio de Sanidad, Consumo y Bienestar Social, 2018).

Al igual que en otros países, con independencia de la tipología de consumo (global, de atracón o problemática) los datos de la ENSE-2017, muestran diferencias en las prevalencias de consumo entre hombres y mujeres tanto para España como para Galicia, siendo estas siempre más altas en los hombres (Ministerio de Sanidad, Consumo y Bienestar Social, 2018).

A pesar de que se dispone de información derivada de la ENSE sobre la prevalencia de consumo de alcohol en la población adulta de Galicia, el tamaño muestral no permite disponer de estimaciones precisas cuando se realizan análisis detallados por regiones en función de variables como el sexo y la edad. Esta información es necesaria, desde el ámbito de trabajo de la Salud Pública, para planificar y evaluar distintas intervenciones orientadas tanto a la prevención primaria como secundaria y terciaria del consumo de alcohol. Estas acciones se definen en función de las diferentes tipologías de consumo, y los bebedores problemáticos constituyen un grupo de especial interés, tanto desde el punto de vista epidemiológico como clínico (Carvalho, Heilig, Perez, Probst y Rehm, 2019). A pesar de que el consumo problemático de alcohol se concentra en los hombres, en los últimos años se ha observado un descenso en las diferencias de consumo entre hombres y mujeres (Organización Mundial de la Salud, 2018), por lo que analizar este problema de salud desde una perspectiva de género es fundamental.

Los objetivos de este trabajo fueron estimar la prevalencia de consumo de alcohol diario, esporádico, ocasional, intensivo y problemático en el último mes e identificar qué variables sociodemográficas, de estado de salud o conductas están relacionadas con un consumo problemático de alcohol en Galicia teniendo en cuenta la perspectiva de género.

\section{Método}

En el año 2005, se implantó en Galicia el Sistema de Información sobre Conductas de Riesgo para la salud (SICRI), que se fundamenta en la realización de estudios transversales mediante encuestas entre la población de 16 años y más residente en Galicia. Las encuestas del SICRI tienen periodicidad casi anual y se realizan por teléfono mediante un sistema CATI (Computed-assisted telephone interview). Entre los objetivos del SICRI está estimar la prevalencia poblacional de diferentes factores de riesgo para la salud.

La encuesta realizada en 2017 (SICRI-2017) se diseñó para garantizar la representatividad de la población definida en función del sexo y en cuatro grupos de edad (16-24, 25-44, 45-64 y 65 años y más). El tamaño de muestra se estableció teniendo en cuenta una prevalencia del $50 \%$ y un error absoluto del 3,5\%. Para cada grupo definido por sexo y edad, se estimó un tamaño teórico de 980 entrevistas, lo que supone un tamaño de muestra global de 7.840 entrevistas. El muestreo fue aleatorio estratificado y se empleó como marco de muestreo la base de datos de la Tarjeta Sanitaria que tiene una cobertura aproximada del $97 \%$ de la población residente en Galicia. El trabajo de campo se llevó a cabo entre enero y diciembre de 2017.

En el cuestionario del SICRI-2017 se incluyeron diferentes preguntas con el objetivo de estimar la prevalencia y caracterizar el consumo de alcohol a nivel poblacional. En relación con el consumo de alcohol en las 4 últimas semanas, se define como bebedor diario a la persona que declara haber bebido a diario alcohol; bebedor esporádico a la que declara haber bebido alcohol todas o casi todas las semanas, pero no a diario; bebedor ocasional a la que dice haber bebido alcohol algún día de alguna de las semanas y 
nunca bebedor a la que declara no haber bebido alcohol. La variable consumo global o consumidor de alcohol hace referencia a la prevalencia conjunta de bebedores diarios, esporádicos y ocasionales. A las personas que declararon haber bebido alcohol las 4 semanas previas a la entrevista, se les preguntó por la frecuencia de consumo de 6 o más bebidas alcohólicas en el mismo día, que se define como consumo intensivo o de atracón.

Se incluyeron las 10 preguntas que forman el cuestionario Alcohol Use Disorders Identification Test (AUDIT). La puntuación total del AUDIT varía entre 0 y 40 puntos; una puntuación $\geq 8$ ( 7 en las mujeres y en los mayores de 64 años) se considera indicativa de un consumo problemático de alcohol (Babor, Higgins-Biddle, Saunders y Monteiro, 2001; Saunders, Aasland, Babor, de la Fuente y Grant, 1993).

Para caracterizar a los bebedores con consumo problemático de alcohol se ajustaron dos modelos de regresión logística multivariante, uno para hombres y otro para mujeres. La variable dependiente fue tener consumo problemático de alcohol y como explicativas se incluyeron variables sociodemográficas, de estado de salud y de conductas relacionadas con la salud. Las variables explicativas incluidas fueron aquellas que se asociaron significativamente con la variable dependiente $(p<0,1)$ en un análisis bivariante previo. En el modelo final se mantuvieron las variables con $\mathrm{p}<0,05$, y a partir de ese modelo se calcularon odds ratios (OR) ajustadas de tener consumo problemático de alcohol.

Las variables sociodemográficas analizadas fueron: grupo de edad (16-24, 25-44, 45-64 y 65 años y más); país de nacimiento (España-otro país); ámbito de residencia (rural, semiurbano y urbano); situación laboral en el momento de la encuesta (trabaja, en paro o sin trabajo, labores del hogar, pensionista, estudiante u otra situación); nivel de estudios (básicos: educación primaria o menos, medios: educación secundaria y superiores: universitarios); y convivencia en pareja (si-no). Como variables de estado de salud se analizaron la autopercepción del estado de salud en el momento de la encuesta (muy bueno, bueno, normal y malo); el estado ponderal (bajo peso, peso normal, sobrepeso y obesidad) valorado a partir de la información autodeclarada de peso y talla; y la autopercepción del peso (gordo, peso adecuado y delgado). Finalmente, las variables de conductas fueron el consumo de tabaco y cannabis (fumador y no fumador) en el momento de la encuesta. El análisis se realizó de forma independiente para hombres y mujeres utilizando Stata v14.2, y en los cálculos se tuvo en cuenta el diseño de la muestra.

La aprobación ética por parte del Comité de Ética de Galicia no fue necesaria porque este estudio fue voluntario y anónimo, garantizando la total confidencialidad. El estudio se realizó mediante entrevistas telefónicas y, en consecuencia, el acuerdo para participar implicaba el consentimiento.
Información detallada del diseño y otros resultados del SICRI-2017 están disponible en la página web de la Dirección General de Salud Pública [https://www.sergas.es/ saude-publica/ SICRI-Sistema-Información-sobre-Conductas-de-Risco].

\section{Resultados}

Se recogió información de 7.841 personas de 16 y más años, siendo la tasa de respuesta del estudio del $78 \%$. En relación al estado de salud y estilos de vida, el 37,9\% de la población gallega de 16 y más años autopercibía su estado de salud como bueno, el 55,1\% tenían sobrepeso u obesidad, el 19,7\% eran fumadores y el 0,9\% tenían un uso problemático de internet. Las características de hombres y mujeres por separado se presentan en la tabla 1 .

El 58,6\% de la población gallega de 16 años y más consumió alcohol en el último mes, el 18,8\% a diario y el 23,4\% de modo ocasional (Tabla 2). En global, la prevalencia de consumo de alcohol se estimó en el 72,8\% en los hombres y en el $45,8 \%$ en las mujeres.

Las prevalencias de consumo diario y ocasional fueron más altas entre los hombres [razón de prevalencias (RP) hombre/mujer: 2,7 para consumo diario y 1,7 para ocasional] (Tabla 2).

En los hombres, la prevalencia de los consumidores de alcohol aumentó con la edad hasta los 45-64 años, que es el grupo con la prevalencia máxima $(77,4 \%)$. En las mujeres, se observó la prevalencia máxima en el grupo de 16 a 24 años $(59,6 \%)$. En cuanto al consumo diario, la prevalencia siguió una tendencia creciente con la edad, tanto en hombres como en mujeres, siendo a cualquier edad más alta entre los hombres (Figura 1).

Entre los gallegos que beben alcohol, se estimó un consumo medio de 1,65 bebidas alcohólicas por día de consumo. Los hombres consumieron en promedio 0,5 consumiciones más que las mujeres los días que beben alcohol, siendo el consumo medio de bebidas alcohólicas de 1,85 en los hombres y de 1,35 en las mujeres. Los consumidores ocasionales, con independencia del sexo, declararon tomar 3 bebidas o más en un día cualquiera de consumo (Tabla 3).

La prevalencia de consumo intensivo más alta se observó en el grupo de 16-24 años, especialmente en los hombres, y siguió una tendencia decreciente con la edad en ambos sexos, siendo en cualquier edad más alta en los hombres (Figura 2). Entre los 25-44 años la prevalencia en hombres triplicó a la de las mujeres (RP hombre/mujer: 3,0).

La prevalencia de consumo problemático de alcohol fue del 3,4\% en la población y del 5,8\% entre los consumidores de alcohol. La prevalencia más alta entre los consumidores de alcohol se observó en los hombres $(8,0 \%)$ y, en función del grupo de edad, entre los 16-24 años (17,5\%) (Tabla 4). 
Tabla 1. Características de los hombres y mujeres gallegos de 16 años y más.

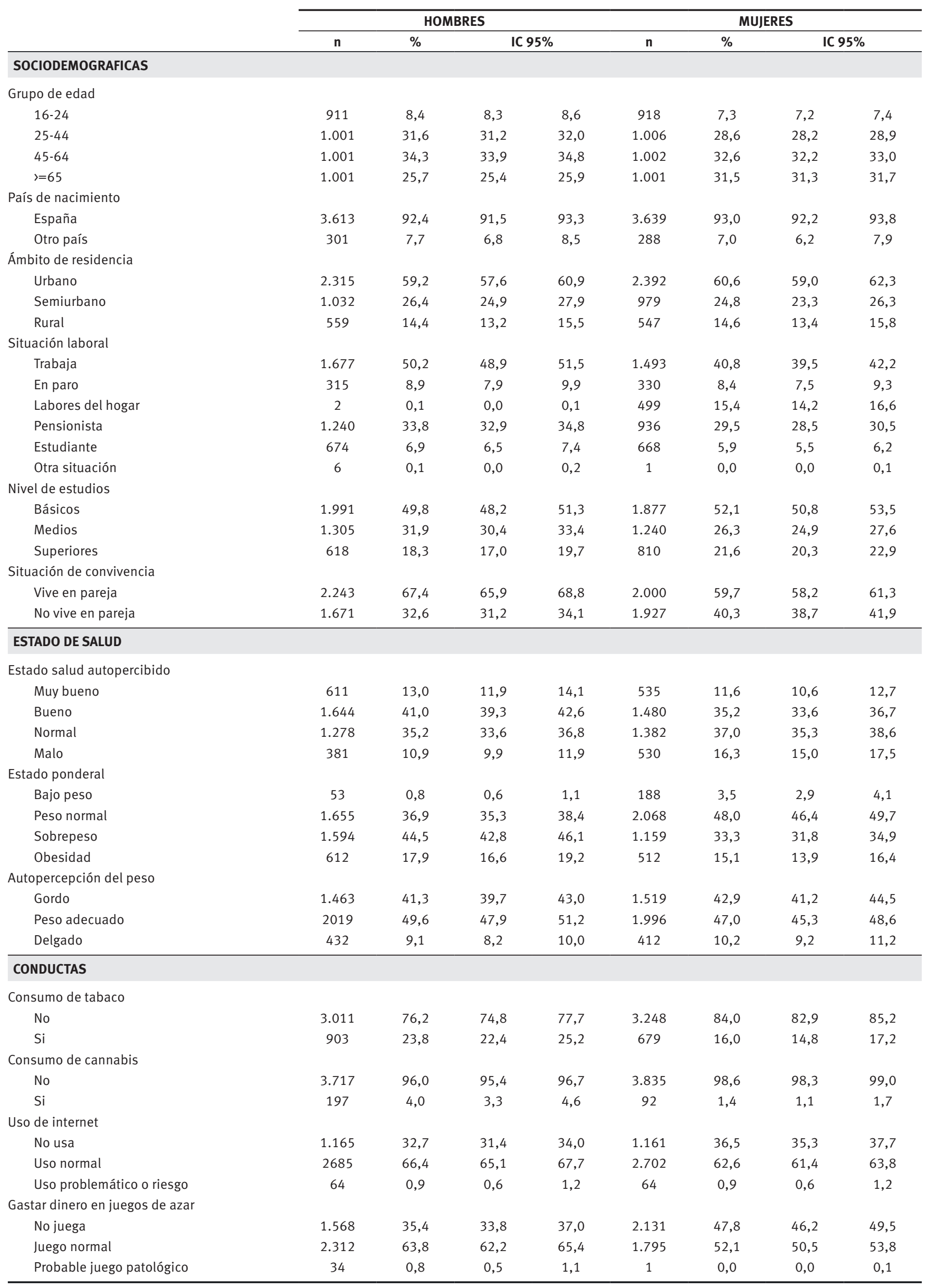


Tabla 2. Prevalencia de consumo de alcohol en la población gallega de 16 años y más.

\begin{tabular}{|c|c|c|c|c|c|c|c|c|c|c|c|c|}
\hline \multirow[b]{3}{*}{ Nunca } & \multicolumn{4}{|c|}{ TOTAL } & \multicolumn{4}{|c|}{ HOMBRES } & \multicolumn{4}{|c|}{ MUJERES } \\
\hline & \multirow{2}{*}{$\frac{\mathbf{n}}{3.132}$} & \multirow{2}{*}{$\begin{array}{c}\% \\
41,4\end{array}$} & \multicolumn{2}{|c|}{ IC $95 \%$} & \multirow{2}{*}{$\frac{\mathbf{n}}{1.105}$} & \multirow{2}{*}{$\begin{array}{c}\% \\
27,2\end{array}$} & \multicolumn{2}{|c|}{ IC $95 \%$} & \multirow{2}{*}{$\begin{array}{c}\mathbf{n} \\
2.027\end{array}$} & \multirow{2}{*}{\begin{tabular}{c|}
$\%$ \\
54,3
\end{tabular}} & \multicolumn{2}{|c|}{ IC $95 \%$} \\
\hline & & & 40,3 & 42,5 & & & 25,8 & 28,7 & & & 52,6 & 55,9 \\
\hline Esporádico & 1.428 & 16,4 & 15,6 & 17,3 & 639 & 14,9 & 13,7 & 16,0 & 789 & 17,9 & 16,6 & 19,1 \\
\hline Ocasional & 1.998 & 23,4 & 22,4 & 24,4 & 1.214 & 29,8 & 28,3 & 31,3 & 784 & 17,6 & 16,3 & 18,8 \\
\hline Diario & 1.283 & 18,8 & 17,9 & 19,7 & 956 & 28,2 & 26,7 & 29,6 & 327 & 10,3 & 9,3 & 11,3 \\
\hline
\end{tabular}

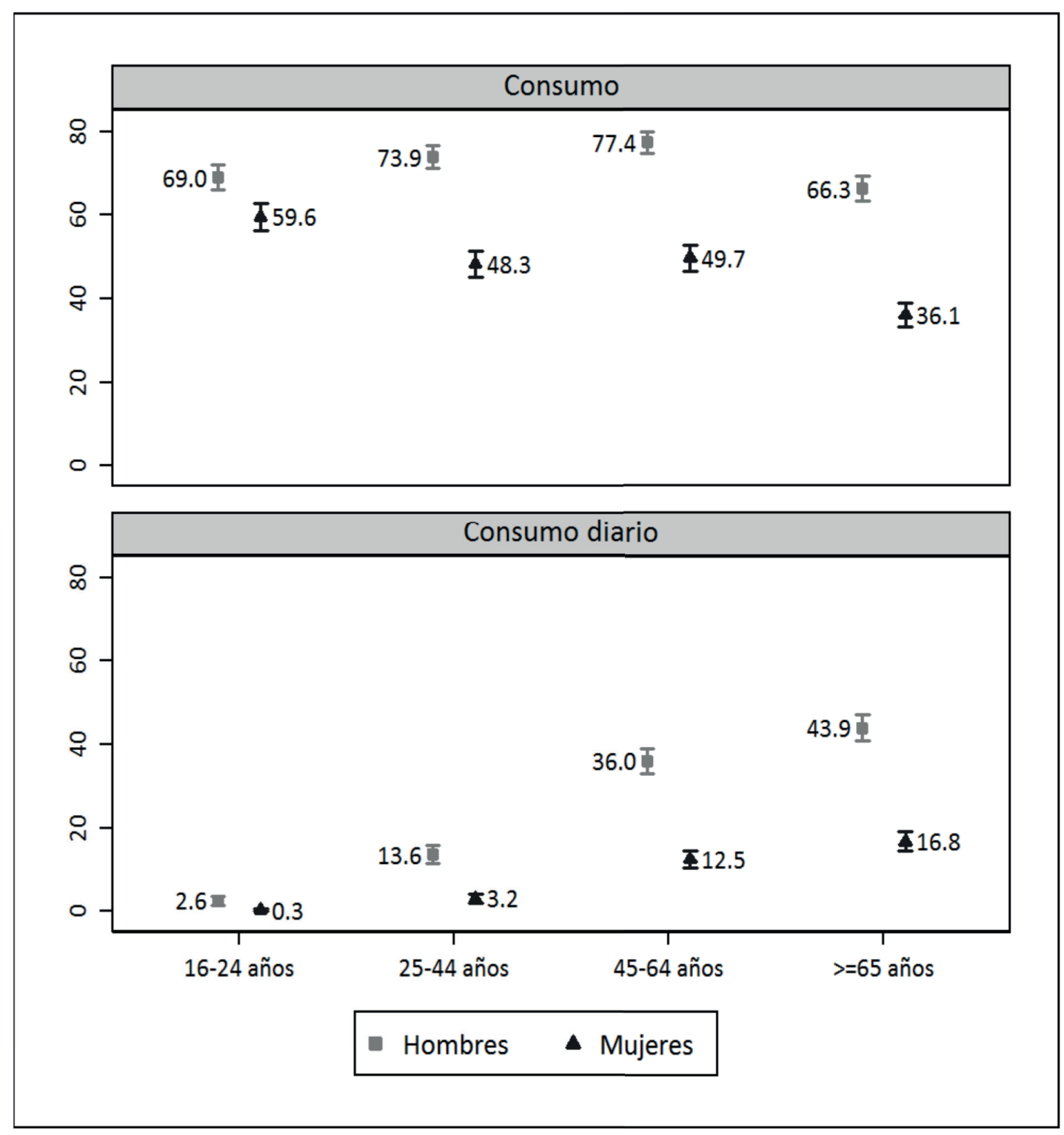

Figura 1. Prevalencia de consumo (diario, esporádico y ocasional) y consumo diario de alcohol en función del sexo (hombres y mujeres) y del grupo de edad (16-24, 25-44, 45-64, 65 años y más) en la población gallega.

Tener menos de 25 años, no vivir en pareja, autopercibirse como una persona "gorda" y ser fumador de tabaco o cannabis son variables que aumentan la probabilidad de tener un consumo problemático de alcohol tanto en hombres como en mujeres. En el caso de los hombres tener un nivel de estudios básicos o medios y valorar su estado de salud como "muy bueno" o "bueno/normal" también son variables que aumentan la probabilidad de consumo problemático. En las mujeres no se identifica ninguna variable adicional (Figuras 3 y 4 ). 
Tabla 3. Distribución del número de consumiciones que toman los consumidores en un día de consumo.

\begin{tabular}{|c|c|c|c|c|c|c|c|c|c|c|c|c|}
\hline \multirow{3}{*}{ Todos los consumidores } & \multicolumn{4}{|c|}{ TOTAL } & \multicolumn{4}{|c|}{ HOMBRES } & \multicolumn{4}{|c|}{ MUJERES } \\
\hline & $\mathbf{n}$ & $\%$ & \multicolumn{2}{|c|}{ IC $95 \%$} & $\mathbf{n}$ & $\%$ & \multicolumn{2}{|c|}{ IC $95 \%$} & $\mathbf{n}$ & $\%$ & \multicolumn{2}{|c|}{ IC $95 \%$} \\
\hline & & & & & & & & & & & & \\
\hline Una o menos & 2.345 & 55,6 & 54,1 & 57,0 & 1.231 & 47,3 & 45,3 & 49,2 & 1.114 & 67,6 & 65,5 & 69,7 \\
\hline Dos & 1.504 & 30,9 & 29,5 & 32,3 & 975 & 35,6 & 33,7 & 37,5 & 529 & 24,1 & 22,1 & 26,1 \\
\hline Tres o más & 860 & 13,6 & 12,6 & 14,5 & 603 & 17,2 & 15,7 & 18,6 & 257 & 8,3 & 7,2 & 9,5 \\
\hline \multicolumn{13}{|l|}{ Esporádicos } \\
\hline Una o menos & 794 & 63,8 & 61,1 & 66,5 & 331 & 58,2 & 54,0 & 62,4 & 463 & 68,0 & 64,5 & 71,4 \\
\hline Dos & 411 & 25,6 & 23,1 & 28,1 & 182 & 27,6 & 23,8 & 31,4 & 229 & 24,1 & 20,9 & 27,3 \\
\hline Tres o más & 223 & 10,7 & 9,0 & 12,3 & 126 & 14,2 & 11,4 & 17,0 & 97 & 8,0 & 6,1 & 9,8 \\
\hline \multicolumn{13}{|l|}{ Ocasionales } \\
\hline Una o menos & 814 & 47,7 & 45,3 & 50,1 & 431 & 40,5 & 37,5 & 43,5 & 383 & 58,7 & 55,0 & 62,5 \\
\hline Dos & 688 & 34,4 & 32,1 & 36,7 & 441 & 38,2 & 35,2 & 41,2 & 247 & 28,6 & 25,2 & 32,1 \\
\hline Tres o más & 496 & 17,9 & 16,2 & 19,7 & 342 & 21,4 & 19,0 & 23,7 & 154 & 12,6 & 10,3 & 15,0 \\
\hline \multicolumn{13}{|l|}{ Diarios } \\
\hline Una o menos & 737 & 58,3 & 55,6 & 61,0 & 469 & 48,7 & 45,5 & 51,9 & 268 & 82,2 & 78,1 & 86,4 \\
\hline Dos & 405 & 31,1 & 28,5 & 33,6 & 352 & 37,0 & 33,9 & 40,1 & 53 & 16,2 & 12,2 & 20,3 \\
\hline Tres o más & 141 & 10,6 & 8,9 & 12,3 & 135 & 14,3 & 12,0 & 16,6 & 6 & 1,6 & 0,3 & 2,8 \\
\hline
\end{tabular}

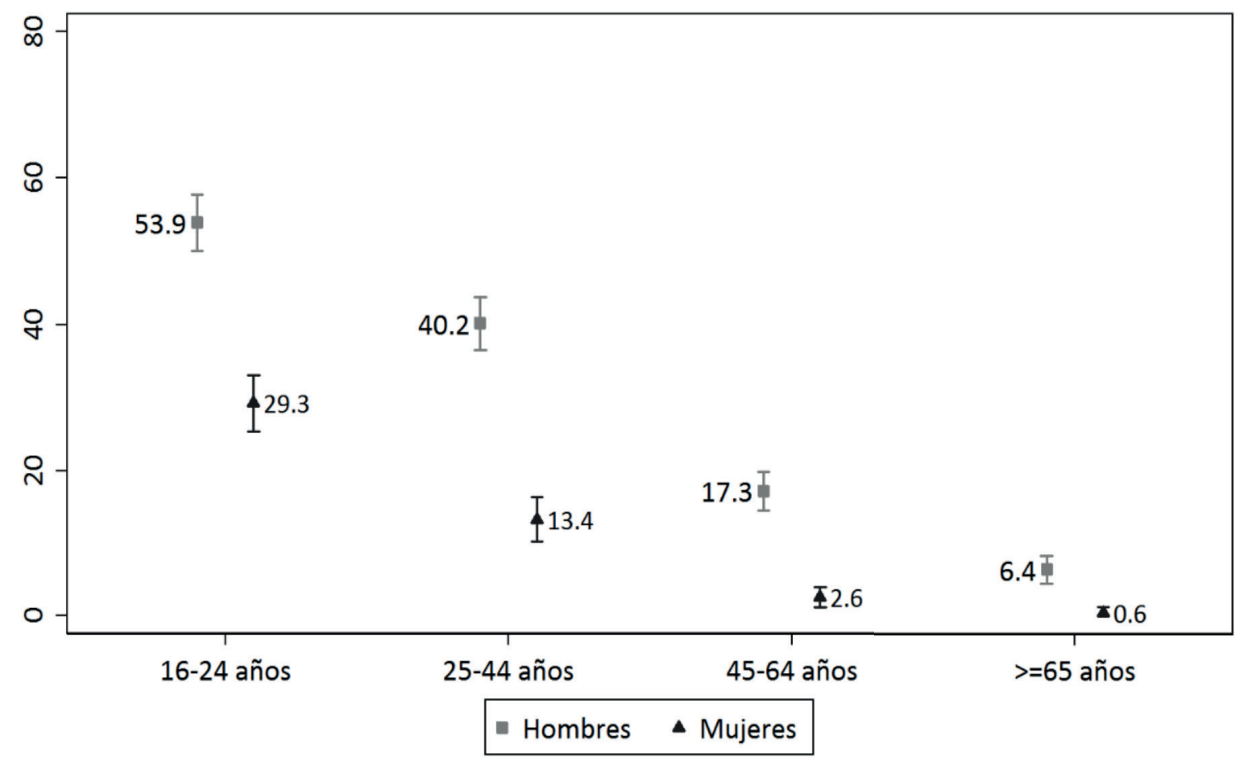

Figura 2. Prevalencia de consumo intensivo de alcohol definido como consumir 6 o más bebidas alcohólicas en el mismo día, en función del sexo (hombres y mujeres) y del grupo de edad (16-24, 25-44, 45-64, 65 años y más) en la población gallega.

Tabla 4. Prevalencia de consumo problemático de alcohol en la población gallega (en la población) y en la población que consume alcohol (en los consumidores) por grupo de edad (16-24, 25-44, 45-64, 65 años y más) en total, en hombres y en mujeres.

\begin{tabular}{|c|c|c|c|c|c|c|c|c|c|c|c|c|}
\hline & \multirow{2}{*}{\multicolumn{4}{|c|}{ TOTAL }} & & & & & & & & \\
\hline & & & & & \multicolumn{4}{|c|}{ HOMBRES } & \multicolumn{4}{|c|}{ MUJERES } \\
\hline & $\mathbf{n}$ & $\%$ & \multicolumn{2}{|c|}{ IC $95 \%$} & $\mathbf{n}$ & $\%$ & \multicolumn{2}{|c|}{ IC $95 \%$} & \multirow[t]{2}{*}{$\mathbf{n}$} & \multirow[t]{2}{*}{$\%$} & \multicolumn{2}{|c|}{ IC 95\% } \\
\hline & & & & & & & & & & & \multicolumn{2}{|c|}{ En la población } \\
\hline $16-24$ & 1.829 & 11,3 & 9,8 & 12,7 & 911 & 15,4 & 13,0 & 17,7 & 918 & 7,0 & 5,3 & 8,6 \\
\hline $25-44$ & 2.007 & 4,4 & 3,5 & 5,2 & 1.001 & 7,6 & 5,9 & 9,2 & 1.006 & 1,1 & 0,5 & 1,7 \\
\hline $45-64$ & 2.003 & 2,3 & 1,7 & 3,0 & 1.001 & 4,0 & 2,8 & 5,2 & 1.002 & 0,7 & 0,2 & 1,2 \\
\hline$>=65$ & 2.002 & 1,6 & 1,1 & 2,1 & 1.001 & 3,0 & 2,0 & 4,1 & 1.001 & 0,5 & 0,1 & 0,9 \\
\hline Total & 7.841 & 3,4 & 3,0 & 3,8 & 3.914 & 5,8 & 5,1 & 6,6 & 3.927 & 1,2 & 0,9 & 1,5 \\
\hline \multicolumn{13}{|c|}{ En los consumidores } \\
\hline $16-24$ & 1.176 & 17,5 & 15,3 & 19,7 & 629 & 22,3 & 19,0 & 25,5 & 547 & 11,7 & 9,0 & 14,4 \\
\hline $25-44$ & 1.236 & 7,1 & 5,7 & 8,6 & 742 & 10,3 & 8,1 & 12,5 & 494 & 2,3 & 0,9 & 3,6 \\
\hline $45-64$ & 1.273 & 3,6 & 2,6 & 4,7 & 775 & 5,2 & 3,6 & 6,7 & 498 & 1,4 & 0,4 & 2,4 \\
\hline$\rangle=65$ & 1.024 & 3,2 & 2,2 & 4,3 & 663 & 4,5 & 3,0 & 6,1 & 361 & 1,4 & 0,2 & 2,6 \\
\hline Total & 4.709 & 5,8 & 5,2 & 6,5 & 2.809 & 8,0 & 7,0 & 9,0 & 1.900 & 2,6 & 2,0 & 3,3 \\
\hline
\end{tabular}




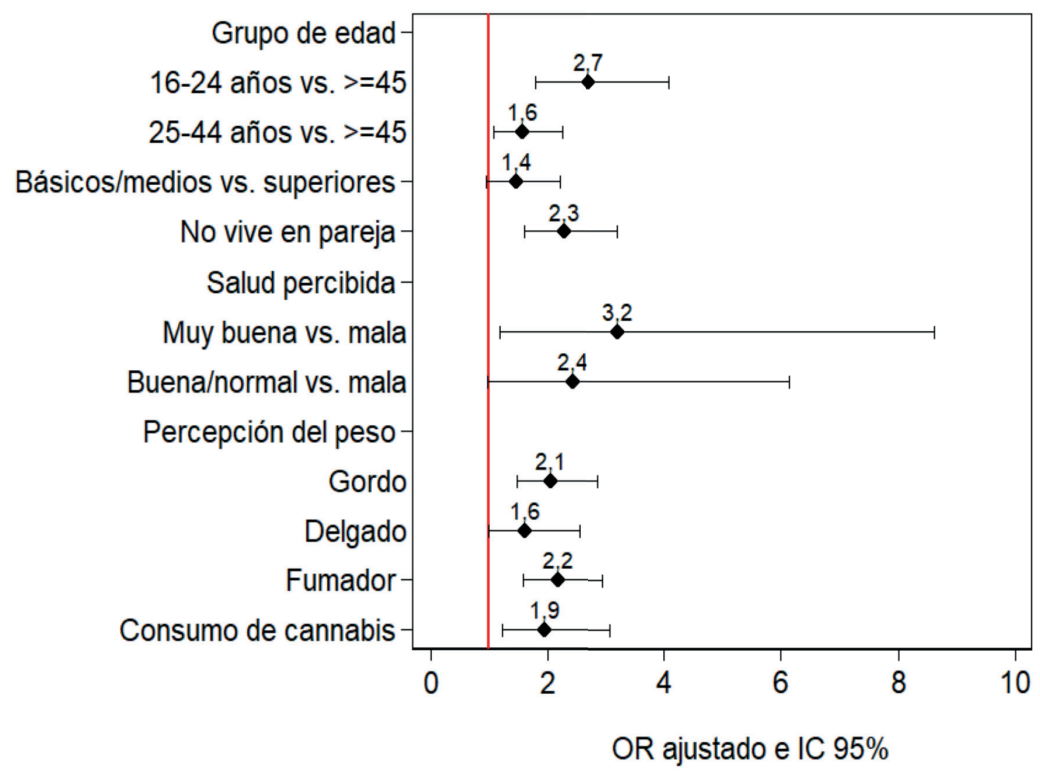

Figura 3. Características que se asocian con el consumo problemático de alcohol en hombres.

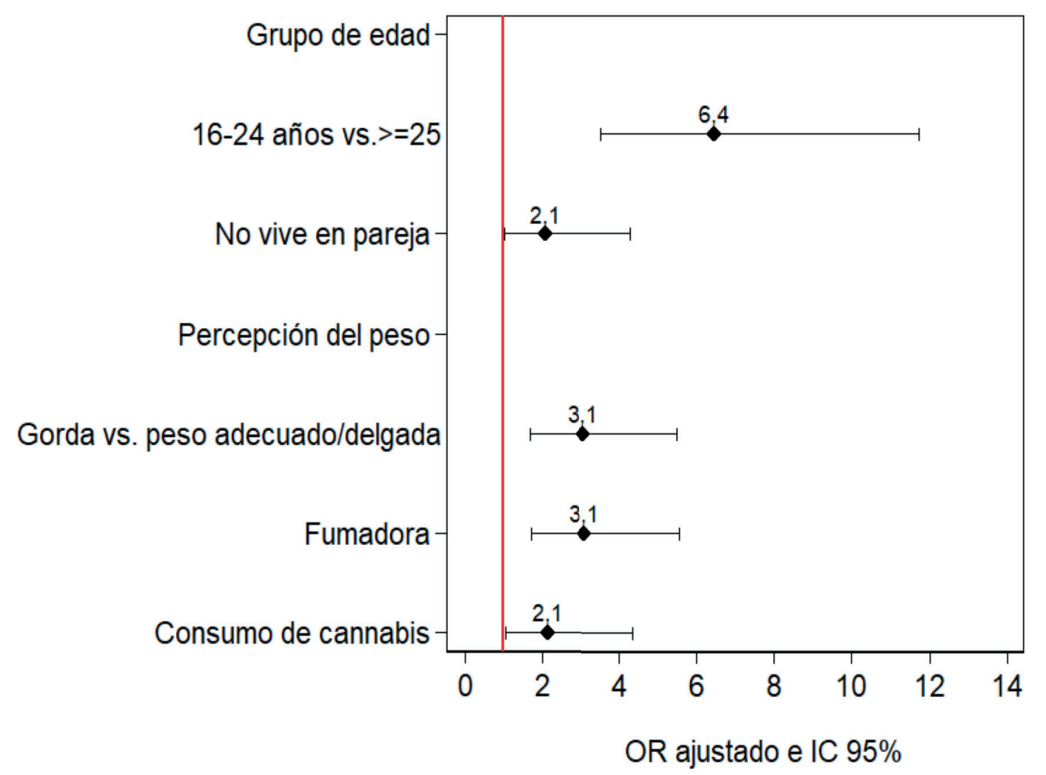

Figura 4. Características que se asocian con el consumo problemático de alcohol en mujeres.

\section{Discusión}

El consumo de alcohol presenta una alta prevalencia en Galicia, identificándose diferencias en los patrones de consumo en función del sexo y grupo de edad. Los hombres, a cualquier edad y con independencia de la tipología de consumo, tienen prevalencias más altas que las mujeres.

En general, los resultados obtenidos en este estudio están en la línea de los datos publicados en la ENSE-2017 para población general española de edad comparable. Ambos estudios estiman prevalencias similares y diferencias en función del sexo, siendo los hombres los que de forma sistemática presentan prevalencias más altas. Las diferencias en función del sexo concuerdan con lo que se observa en otros estudios internacionales, donde las prevalencias más altas también se observan en los hombres, aunque la diferencia por sexo varía en función del país (Organización Mundial de la Salud, 2018). La influencia de los factores sociales y culturales de cada país puede explicar esta variabilidad. En sociedades donde existe la creencia de que el consumo de alcohol en las mujeres puede afectar a su rol en la sociedad se produce una mayor restricción en su consumo. Por otro lado, en sociedades donde la igualdad de género ha aumentado, se observa un incremento en la adopción de conductas perjudiciales por parte de las muje- 
res que tradicionalmente eran comunes entre los hombres, como es el consumo de alcohol (Bosque-Prous et al., 2015; Galán, González y Valencia-Martín, 2014; White, 2020).

En relación con el consumo intensivo de alcohol, también conocido como de atracón o binge drinking, hay que tener en cuenta las diferencias en su definición entre estudios, tanto en relación con el número de bebidas alcohólicas ingeridas como con el marco temporal. A pesar de esto, los resultados de nuestro estudio coinciden con los de estudios previos realizados en España, Estados Unidos o Francia, al identificar que el consumo intensivo de alcohol se da con mayor frecuencia al final de la adolescencia o comienzo de la vida adulta y entre los hombres (Galán et al., 2020; Krieger, Young, Anthenien y Neighbors, 2018; Molina y Nelson, 2018; Rodríguez Muñoz, Carmona Torres, Hidalgo Lopezosa, Cobo Cuenca y Rodríguez Borrego, 2019; Soler-Vila et al., 2014; Tavolacci et al., 2016). A pesar de que las mujeres tienen un menor consumo binge drinking que los hombres, este tipo de consumo está aumentando entre ellas y las diferencias entre hombres y mujeres jóvenes tienden a desaparecer (Wilsnack, Wilsnack, Gmel y Kantor, 2018). Una de las cuestiones que puede explicar que las personas más jóvenes tengan un mayor consumo intensivo de alcohol es la asociación demostrada entre el binge drinking y el fenómeno "botellón”, entendido como un grupo de personas, mayoritariamente jóvenes, que se reúnen para consumir alcohol en espacios públicos al aire libre. En el "botellón" se crea una situación donde factores como la presión por parte del grupo para beber alcohol, una mayor accesibilidad a bebidas más baratas y la creencia de efectos positivos, como que facilitará las relaciones sociales, pueden influir en la realización de un consumo intensivo de alcohol (Llamosas-Falcón, Manthey y Rehm, 2020; Teixidó-Compañó et al., 2019).

El consumo problemático de alcohol es un problema de Salud Pública que afecta de forma desigual a la población mundial (Carvalho et al., 2019). España es uno de los países europeos con la prevalencia más baja (Carvalho et al., 2019) y aun así afecta al 5,6\% de la población menor de 65 años (Observatorio Español de las Dogas y las Adicciones, 2021). En Galicia esta prevalencia se ha estimado en el 3,4\% observándose, al igual que en otros estudios, una mayor prevalencia entre hombres (Bosque-Prous et al., 2015). Además, resulta preocupante que en Galicia el grupo de edad con mayor prevalencia de consumo problemático se sitúe entre los 16 y 24 años y que sea en este grupo donde la razón de prevalencias en función del sexo sea la más baja de las observadas en los diferentes grupos de edad. En un estudio donde se analizaron las diferencias de género entre países europeos en el consumo problemático de alcohol, se observó que este tipo de consumo es mayoritario entre los hombres y sobre todo en los países de Europa del Este. Sin embargo, se observó que, en aquellos países con mayor igualdad entre hombres y mujeres, las diferencias en este tipo de consumo eran menores a causa de una mayor prevalencia de consumo problemático de alcohol por parte de las mujeres (Bosque-Prous et al., 2015).

Son escasos los estudios en los que se han analizado las características asociadas al consumo problemático de alcohol en población adulta en función del sexo. En nuestro trabajo se ha observado que los hombres y mujeres gallegas con un consumo problemático de alcohol presentan características comunes en relación con la edad, la convivencia en pareja, la percepción del peso y las conductas adictivas relacionadas con el consumo de tabaco y el cannabis. Si bien, el nivel de estudios, variable asociada con el consumo problemático de alcohol por otros autores, solo parece influir en los hombres.

$\mathrm{Al}$ igual que en un estudio anterior, se ha observado que los hombres y mujeres jóvenes tienen una mayor probabilidad de tener un consumo problemático de alcohol (Skinner y Veilleux, 2016). Tener entre 16 y 24 años fue el mayor determinante de consumo problemático en las mujeres. Al igual que en otros estudios, en nuestro estudio se observó que las conductas de riesgo se relacionan entre ellas, así la probabilidad de consumo problemático de alcohol es mayor entre los fumadores/as de tabaco o cannabis (Berge, Håkansson y Berglund, 2014; Bosque-Prous et al., 2017; Park y Kim, 2019), sobre todo, entre las mujeres. En nuestro estudio la probabilidad de consumo problemático de alcohol aumenta entre las personas que viven sin pareja. Estudios previos habían identificado esta relación en los hombres (Bosque-Prous et al., 2017; Park et al., 2019), sin embargo, en las mujeres hay discrepancias (Bosque-Prous et al., 2017; Teixidó-Compañó et al., 2018). El nivel de estudios es una variable tradicionalmente relacionada con el consumo problemático de alcohol (Bosque-Prous et al., 2017; Park et al., 2019; Teixidó-Compañó et al., 2018). La relación que se observa entre el nivel de estudios y el consumo problemático de alcohol difiere entre hombres y mujeres. En los hombres, el consumo problemático se asocia con el bajo nivel de estudios. En las mujeres, esta relación no está establecida y algunos autores apuntan a que el consumo problemático se asocia a un mayor nivel de estudios (Bosque-Prous et al., 2017; Devaux y Sassi, 2016; Park et al. 2019). En nuestro trabajo, al igual que en otro estudio realizado en España (Teixidó-Compañó et al., 2018), no se encontró relación entre el nivel de estudios y el consumo problemático de alcohol en las mujeres.

Que sepamos esta es la primera vez que se valora la influencia de la autopercepción del peso en relación con el consumo problemático de alcohol. Tanto hombres como mujeres que se perciben como personas "gordas" tienen una mayor probabilidad de tener un consumo problemático de alcohol. En el caso de los hombres, considerarse una persona "delgada" también aumenta la probabilidad de consumo problemático, mientras que en las mujeres lo disminuye. En relación con el estado de salud autopercibi- 
do, resultados previos ya habían observado que, entre los hombres, percibir su estado de salud como bueno o muy bueno aumenta la probabilidad de tener un consumo problemático de alcohol (Bosque-Prous et al., 2017).

Entre las limitaciones de este estudio destaca la autodeclaración telefónica del consumo de alcohol que no permite realizar una medición detallada de las unidades de bebida consumidas por la población. La autodeclaración de conductas con connotaciones sociales negativas está afectada por un sesgo de deseabilidad social, que apunta hacia la ocultación de la conducta y por lo tanto a la infraestimación de la prevalencia real del consumo. En este caso este sesgo podría afectar especialmente a la prevalencia de consumo problemático de alcohol, por lo que las estimaciones aquí presentadas deben tomarse con cautela (Staudt et al., 2019; Valencia-Martín, González y Galán, 2014). La mayor fortaleza de este estudio es su tamaño muestral, que permite llevar a cabo un análisis detallado de la tipología de consumo de alcohol a nivel de una comunidad autónoma con datos representativos por sexo y grupo de edad, aspecto que las encuestas realizadas a nivel nacional no permiten realizar. Otra de las fortalezas, es que la muestra está equidistribuída durante los 12 meses del año, evitando así la influencia de la estacionalidad del consumo de alcohol.

Este estudio objetiva la realidad del consumo de alcohol entre los adultos gallegos, estimando prevalencias elevadas en una conducta que pone en riesgo la salud y tiene consecuencias a nivel personal, familiar, laboral y social. Las acciones preventivas desde el sistema sanitario deben ser prioritarias tanto orientadas a la prevención primaria, secundaria como terciaria. Es importante seguir monitorizando el consumo de alcohol para detectar cambios en los patrones, comportamientos y tendencias en la población.

\section{Conflicto de intereses}

Todos los autores declaran no tener conflictos de intereses.

\section{Referencias}

Babor, T., Higgins-Biddle, J., Saunders, J. y Monteiro, M. (2001). Cuestionario de Identificación de los Trastornos debidos al Consumo de Alcohol: AUDIT. Recuperado de https://www.who.int/substance_abuse/activities/en/AUDITmanualSpanish.pdf.

Barrio, P., Reynolds, J., García-Altés, A., Gual, A. y Anderson, P. (2017). Social costs of illegal drugs, alcohol and tobacco in the European Union: A systematic review. Drug and Alcohol Review, 36, 578-588. doi:10.1111/ dar. 12504.

Berge, J., Håkansson, A. y Berglund, M. (2014). Alcohol and drug use in groups of cannabis users: Results from a survey on drug use in the Swedish general popula- tion. The American Journal on Addictions, 23, 272-279. doi:10.1111/j.1521-0391.2014.12097.x.

Bosque-Prous, M., Brugal, M. T., Lima, K. C., Villalbí, J. R., Bartroli, M. y Espelt, A. (2017). Hazardous drinking in people aged 50 years or older: A cross-sectional picture of Europe, 2011-2013. International Journal of Geriatric Psychiatry, 32, 817-828. doi:10.1002/gps.4528.

Bosque-Prous, M., Espelt, A., Borrell, C., Bartroli, M., Guitart, A. M., Villalbí, J. R. y Brugal, M. T. (2015). Gender differences in hazardous drinking among middle-aged in Europe: The role of social context and women's empowerment. European Journal of Public Health, 25, 698705. doi:10.1093/eurpub/cku234.

Carvalho, A. F., Heilig, M., Perez, A., Probst, C. y Rehm, J. (2019). Alcohol use disorders. Lancet, 394, 781-792. doi:10.1016/S0140-6736(19)31775-1.

Devaux, M. y Sassi, F. (2016). Social disparities in hazardous alcohol use: Self-report bias may lead to incorrect estimates. European Journal of Public Health, 26, 129-134. doi:10.1093/eurpub/ckv190.

Galán, I., González, M. J. y Valencia-Martín, J. L. (2014). Patrones de consumo de alcohol en España: Un país en transición. Revista Española de Salud Pública, 88, 529-540. doi:10.4321/S1135-57272014000400007.

Galán, I., Simón, L., Rodríguez-Blázquez, C., Ortiz, C., López-Cuadrado, T. y Merlo, J. (2020). Individual and contextual factors associated with hazardous drinking in Spain: Evidence from a national population-based study. Alcoholism, Clinical and Experimental Research, 44, 2247-2256. doi:10.1111/acer.14451.

GBD 2016 Alcohol Collaborators (2018). Alcohol use and burden for 195 countries and territories, 1990-2016: A systematic analysis for the Global Burden of Disease Study 2016. Lancet, 392, 1015-1035. doi:10.1016/S01406736(18)31310-2.

Krieger, H., Young, C. M., Anthenien, A. M. y Neighbors, C. (2018). The epidemiology of binge drinking among college-age individuals in the United States. Alcohol Research: Current Reviews, 39, 23-30.

Llamosas-Falcón, L., Manthey, J. y Rehm, J. (2020). Cambios en el consumo de alcohol en España de 1990 a 2019. Adicciones. Avance de publicación on-line. doi:10.20882/ adicciones.1400.

Łyszczarz, B. (2019). Production losses associated with alcohol-attributable mortality in the European Union. International Journal of Environmental Research and Public Health, 16, 3536. doi:10.3390/ijerph16193536.

Maisto, S. A. y Saitz, R. (2003). Alcohol use disorders: Screening and diagnosis. The American Journal on Addiction, 12 (Supl. 1), 12-25. doi:10.1111/j.1521-0391.2003. tb00493.x.

Ministerio de Sanidad, Consumo y Bienestar Social. (2018). Encuesta Nacional de Salud de España 2017. Recuperado 
de https://www.mscbs.gob.es/estadEstudios/estadisticas/encuestaNacional/encuesta2017.htm.

Ministerio de Sanidad, Consumo y Bienestar Social. (2020). Limites de consumo de bajo riesgo de alcohol. Actualización del riesgo relacionado con los niveles de consumo de alcohol, el patrón de consumo y el tipo de bebida. Recuperado de https://www.mscbs.gob.es/profesionales/saludPublica/prevPromocion/Prevencion/alcohol/docs/Limites_Consumo_Bajo_Riesgo_Alcohol_Actualizacion.pdf.

Molina, P. E. y Nelson, S. (2018). Binge drinking's effects on the body. Alcohol Research: Current Reviews, 39, 99-109.

Observatorio Español de las Drogas y las Adicciones. (2021). Encuesta sobre alcohol y otras drogas en España, EDADES 2019/2020. Recuperado de https://pnsd.sanidad. gob.es/profesionales/sistemasInformacion/sistemaInformacion/encuestas_EDADES.htm.

Organización Mundial de la Salud. (2018). Global status report on alcohol and health 2018. Recuperado de https:// apps.who.int/iris/bitstream/handle/10665/274603/9 789241565639-eng.pdf?ua=1.

Park, E. y Kim, Y. S. (2019). Gender differences in harmful use of alcohol among Korean adults. Osong Public Health and Research Perspectives, 10, 205-214. doi:10.24171/j. phrp.2019.10.4.02.

Peacock, A., Leung, J., Larney, S., Colledge, S., Hickman, M., Rehm, J.,... Degenhardt, L. (2018). Global statistics on alcohol, tobacco and illicit drug use: 2017 status report. Addiction, 113, 1905-1926. doi:10.1111/add.14234.

Pulido, J., Indave-Ruiz, B. I., Colell-Ortega, E., Ruiz-García, M., Bartroli, M. y Barrio, G. (2014). Estudios poblacionales en España sobre daños relacionados con el consumo de alcohol. Revista Española de Salud Pública, 88, 493-513. doi:10.4321/S1135-57272014000400005.

Rehm, J., Gmel Sr, G. E., Gmel, G., Hasan, O., Imtiaz, S., Popova, S.,... Shuper, P. A. (2017). The relationship between different dimensions of alcohol use and the burden of disease-an update. Addiction, 112, 968-1001. doi:10.1111/add.13757.

Rodríguez Muñoz, P. M., Carmona Torres, J. M., Hidalgo Lopezosa, P., Cobo Cuenca, A. I. y Rodríguez Borrego, M. A. (2019). Evolution of alcohol and tobacco consumption in young people in Spain, after the law 42/2010 against smoking: 2011-2014. Adicciones, 31, 274283. doi:10.20882/adicciones.1035.

Sánchez Sánchez, A., Redondo Martín, S., García Vicario, M. I. y Velázquez Miranda, A. (2012). Episodios de urgencia hospitalaria relacionados con el consumo de alcohol en personas de entre 10 y 30 años de edad en Castilla y León durante el período 2003-2010. Revista Española de Salud Pública, 86, 409-417.

Saunders, J. B., Aasland, O. G., Babor, T. F., de la Fuente, J. R. y Grant, M. (1993). Development of the Alcohol Use Disorders Identification Test (AUDIT): WHO collaborative project on early detection of persons with harmful alcohol consumption-II. Addiction, 88, 791-804. doi:10.1111/j.1360-0443.1993.tb02093.x.

Skinner, K. D. y Veilleux, J. C. (2016). The interactive effects of drinking motives, age, and self-criticism in predicting hazardous drinking. Substance Use E्F Misuse, 51, 1342-1352. doi:10.3109/10826084.2016.1168448.

Soler-Vila, H., Galán, I., Valencia-Martín, J. L., León-Muñoz, L. M., Guallar-Castillón, P. y Rodríguez-Artalejo, F. (2014). Binge drinking in Spain, 2008-2010. Alcoholism, Clinical and Experimental Research, 38, 810-819. doi:10.1111/acer.12275.

Staudt, A., Freyer-Adam, J., Meyer, C., Bischof, G., John, U. y Baumann, S. (2019). Does prior recall of past week alcohol use affect screening results for at-risk drinking? Findings from a randomized study. PloS one, 14, e0217595. doi:10.1371/journal.pone.0217595.

Tavolacci, M. P., Boerg, E., Richard, L., Meyrignac, G., Dechelotte, P. y Ladner, J. (2016). Prevalence of binge drinking and associated behaviours among 3286 college students in France. BMC Public Health, 16, 178. doi:10.1186/s12889-016-2863-x.

Teixidó-Compañó, E., Espelt, A., Sordo, L., Bravo, M. J., Sarasa-Renedo, A., Indave, B. I.,... Brugal, M. T. (2018). Differences between men and women in substance use: The role of educational level and employment status. Gaceta Sanitaria, 32, 41-47. doi:10.1016/j.gaceta.2016.12.017.

Teixidó-Compañó, E., Sordo, L., Bosque-Prous, M., Puigcorbé, S., Barrio, G., Brugal, M. T.,... Espelt, A. (2019). Factores individuales y contextuales relacionados con el binge drinking en adolescentes españoles: Un enfoque multinivel. Adicciones, 31, 41-51. doi:10.20882/adicciones.975.

Valencia-Martín, J. L., González, M. J. y Galán, I. (2014). Methodological issues in the measurement of alcohol consumption: The importance of drinking patterns. $R e-$ vista Española de Salud Pública, 88, 433-446. doi:10.4321/ S1135-57272014000400002.

Wilsnack, R. W., Wilsnack, S. C., Gmel, G. y Kantor, L. W. (2018). Gender differences in binge drinking. Alcohol Research: Current Reviews, 39, 57-76.

White, A. M. (2020). Gender differences in the epidemiology of alcohol use and related harms in the United States. Alcohol Research: Current Reviews, 40, 01. doi:10.35946/ arcr.v40.2.01. 